\title{
La sélection des candidats aux études de médecine répond à une problématique de nature éthique avant d'être un problème méthodologique
}

La présente livraison de la revue propose deux contributions relatives à la problématique de la sélection des étudiants en médecine. Les travaux sont issus de deux contextes géographiques différents, le Québec et la France. Au-delà de la nature de leurs questions de recherches respectives et des résultats qu'elles rapportent, ces deux études illustrent aussi de façon éloquente à quel point les enjeux liés à la sélection des étudiants en médecine se posent de façon différente selon les contextes culturels.

Effectué en contexte nord-américain, le travail de Jean-Sébastien Renaud et de ses collaborateurs ${ }^{[1]}$ documente certaines qualités psychométriques des mini-entrevues multiples (MEM). De telles épreuves ont été développées initialement à l'Université de McMaster pour évaluer certaines habiletés dites «non-cognitives » des candidats aux études de médecine. Elles ont été transposées plus récemment dans le cadre de la procédure de sélection qu'utilisent les trois facultés de médecine francophones du Québec sous le format de mini-entrevues multiples francophones intégrées (MEMFI). A partir d'un échantillon d'étudiants inscrits au programme de doctorat en médecine de l'Université Laval, ils montrent que les résultats aux MEMFI sont corrélés à ceux obtenus à certaines épreuves académiques d'évaluation. En dépit de certaines limites, ils considèrent que les MEMFI ont une validité suffisante pour être utilisées en contexte francophone. La préoccupation prioritaire qui est ainsi illustrée est que les dispositifs de sélection des étudiants en médecine devraient avant tout sélectionner des étudiants qui, d'une part, réussiront leurs études mais qui, aussi, sont dotés d'attributs individuels censés renforcer la probabilité qu'ils deviendront des médecins pourvus des qualités professionnelles attendues d'eux, comme par exemple celles qui sont désignées par le cadre de compétences CanMEDS $^{[2]}$ du Collège royal des médecins et chirurgiens du Canada (expert de contenu abouti, démontrant des compétences de gestion, de communication et de collaboration, tout en étant un acteur majeur dans la promotion de la santé, ainsi qu'un professionnel respectueux de l'éthique exerçant de manière érudite).

Marie-Paule Gustin et ses collaborateurs ${ }^{[3]}$ ont examiné pour leur part les approches d'apprentissage qu'adoptent les étudiants inscrits en première année commune des études de santé (PACES) en France, à l'issue de laquelle un concours très sélectif autorise ou non les étudiants à poursuivre leurs études dans l'une ou l'autre des filières de santé (médecine, odontologie, maïeutique, pharmacie, kinésithérapie). Ils ont également cherché à étudier l'impact de ces approches d'apprentissage sur la réussite au concours. Pour ce faire, ils ont administré aux étudiants une version française du questionnaire R-SPQ-2F de Biggs. Leurs résultats montrent que les étudiants qui ont le plus de chance de réussir sont ceux qui s'intéressent à leurs études, tout en se limitant au contenu déclaratif du cours, ce qui documente le fait que la sélectivité du 
concours encourage en partie des approches d'apprentissage en surface, au détriment d'une approche plus en profondeur. De tels résultats étaient en réalité fort prévisibles au regard de ce qui était déjà connu de l'impact général sur les apprentissages d'une mise en compétition excessive des étudiants, telle qu'elle est organisée en France en PACES, au nom d'une conception républicaine -et très élitiste- du mérite et de l'égalité.

Dans tous les pays, le nombre des candidats aux études de médecine -et plus généralement aux études supérieures conduisant à l'exercice de professions de santé- dépasse largement le nombre des places disponibles. Dès lors, se pose inéluctablement le problème du choix des méthodes, des procédés et des épreuves à mettre en œuvre et, corollairement, des critères à prendre en compte pour la sélection des étudiants. Dans ces mêmes colonnes, nous avions déjà consacré un éditorial à ce thème il y a plus d'une dizaine d'années, en soulignant le fait que cette sélection répondait d'abord à une pratique sociale avant d'être un problème strictement pédagogique ${ }^{[4]}$.

La littérature internationale spécialisée en éducation médicale continue à publier régulièrement de nombreuses contributions consacrées à ce thème. Il s'agit, d'une part, de travaux de recherche développés dans le cadre des sciences de l'éducation appliquées à la formation des professionnels de la santé. Dans une perspective d'inspiration positiviste centrée sur les preuves tangibles (evidence-based education), certains cherchent à documenter, par exemple, les qualités métrologiques de telle ou telle procédure. Dans le même courant, d'autres travaux sont des revues méthodiques et systématiques, visant à identifier les effets démontrés du recours à différentes épreuves ${ }^{[5,6,7]}$. En s'appuyant sur des prémisses épistémologiques alternatives, d'autres travaux s'efforcent de mettre à jour des phénomènes plus complexes. Certains, par exemple, dans le cadre d'une analyse de discours d'inspiration Foucaldienne, cherchent à rendre intelligible la généalogie des représentations et des valeurs sociales qui sont en cause dans les différents choix qui sont effectués ${ }^{[8]}$. Il peut s'agir, par ailleurs, de recommandations consensuelles émanant d'organisations internationales en éducation médicale ${ }^{[9]}$, visant à énoncer les caractéristiques d'un «bon » dispositif de sélection, au regard à la fois de critères édumétriques et de principes liés aux exigences de la responsabilité sociale des facultés de médecine ${ }^{[10]}$.

D'une façon générale, au-delà de la diversité de leurs formats et modalités, les dispositifs de sélection des étudiants en médecine poursuivent trois intentions ${ }^{[9]}$. D'un côté, il s'agit de vérifier qu'on laisse s'engager dans des études longues, réputées exigeantes en termes d'efforts académiques à fournir, des étudiants suffisamment outillés pour y réussir. À cet égard, la littérature fait habituellement référence à la nécessité de mesurer des aptitudes dites cognitives et il est désormais largement démontré que les résultats académiques antérieurs, par exemple ceux documentés lors des dernières années des études secondaires, ont une capacité prédictive très robuste de la réussite aux études ${ }^{[7]}$. De l'autre, on s'efforce d'identifier des étudiants qui seraient dotés de qualités personnelles considérées comme des aptitudes souhaitables pour qu'ils deviennent effectivement les «bons » médecins attendus par la société. On fait alors communément référence à des aptitudes qualifiées de non cognitives, ou parfois à des qualités dites humanistes, en désignant par-là, par exemple, l'intégrité, l'intelligence émotionnelle, l'altruisme, l'aptitude au questionnement éthique, ou encore la capacité à travailler en collaboration. La troisième préoccupation, surtout affirmée en contexte nord-américain ou anglo-saxon, vise à élargir l'accès aux études médicales à des étudiants issus d'origines sociales ou culturelles diversifiées, représentatifs de toutes les communautés dont les professionnels de santé ont à prendre soin, avec le souci de minimiser la très forte sélection sociale observée à l'issue des dispositifs de formation les plus élitistes.

De nombreuses tensions parcourent ces différentes intentions.

Ainsi la distinction des aptitudes personnelles selon qu'elles seraient cognitives ou non cognitives doit-elle être tenue aujourd'hui comme éminemment réductrice, au regard des acquis récents des neurosciences, comprises au sens le plus large. Une conception strictement cognitive des processus d'apprentissage n'est en effet aujourd'hui plus tenable ${ }^{[11]}$ et toutes les théories de la décision rationnelle sont 
aujourd'hui remises en cause à la lumière de l'articulation cognition-émotion ${ }^{[12]}$. Par ailleurs, les recherches en cognition sociale illustrent à quel point nos comportements sociaux sont sous l'influence de multiples déterminants liés, par exemple, à l'appartenance à une catégorie sociale, à un groupe professionnel, à une origine particulière, au genre ou encore au rapport de pouvoir auquel on est soumis. Enfin, les aptitudes dites non-cognitives sont souvent mises en lien avec des compétences transversales ou génériques, qui seraient à développer durant le cursus et dont elles devraient être prédictives, alors que l'on sait l'ambiguiité et les impasses pédagogiques de telles compétences transversales ${ }^{[13,14]}$. Il y a par ailleurs une forme de paradoxe dans cette tentation qu'il pourrait y avoir à attendre des étudiants qu'ils démontrent, avant même d'entrer dans des études, des qualités qui sont attendues d'eux à l'issue de la formation, en tant que résultats des apprentissages qu'ils y feront. Certes, on prend garde de préciser qu'il s'agit simplement de prédire le développement de ces qualités mais les modèles conceptuels qui permettent de faire de telles inférences sont en réalité peu robustes.

La manière de documenter la robustesse souhaitable devrait par ailleurs être envisagée dans une perspective épistémologique ouverte. Elle est le plus souvent abordée dans une logique de démonstration expérimentale, adossée elle-même à une perspective psychométrique qui privilégie les qualités métrologiques (validité et fidélité) des procédures de recueil d'indicateurs quantitatifs et qui recourt à l'interprétation statistique. Elle pourrait l'être avec tout autant de légitimité dans une perspective qualitative de validation de processus, en recourant aux critères de rigueur de l' analyse interprétative du paradigme constructiviste, que sont notamment la crédibilité, la fiabilité et la transférabilité. Seul l'ancrage foncier des sciences médicales contemporaines dans le paradigme positiviste conduit à postuler que les décisions à fort enjeu que sont les décisions d'admission dans les études médicales seraient plus rigoureuses lorsqu'elles se fondent sur des mesures. Du reste, dans un parti pris assez radical mais socialement accepté, aux Pays-Bas, une partie de la procédure de sélection repose sur un tirage au sort ${ }^{[15]}$.
En tout état de cause, le choix fait en France de fonder exclusivement la sélection des étudiants sur la mesure d'aptitudes cognitives, dans une logique purement docimologique, en plus des effets rapportés sur les approches d'apprentissage, induit des conséquences importantes en termes de sélection sociale des étudiants autorisés à poursuivre leurs études de médecine. Ainsi, selon une enquête de la Direction de la recherche, des études, de l'évaluation et des statistiques $^{[16]}$, « parmi les inscrits en PACES, un enfant de cadre a deux fois plus de chance qu' un enfant d'ouvrier d'intégrer une deuxième année, cet écart s'élevant même à 2,5 pour les études de médecine, alors que les chances de réussite sont comparables pour le concours de sages-femmes». Les facteurs en sont bien entendus multiples et l'on pourrait notamment y voir, dans une perspective sociologique Bourdieusienne, une conséquence des habitus acquis dans les milieux sociaux favorisés ${ }^{[17]}$. Le fait que, selon l'Association nationale des étudiants en médecine de France, pour mieux se préparer à la compétition et augmenter leurs chances de succès, $66 \%$ des étudiants recourent à des officines de préparations privées dont le coût est de 1500 à 9000 euros par an, y a sans doute aussi sa part. Il faut donc saluer les expérimentations en cours, comme celle conduite à l'Université d'Angers, qui visent à diversifier les procédures d'admission et peut-être, en conséquence, les profils et origines des étudiants sélectionnés ${ }^{[18]}$.

En l'occurrence, presque tous les pays sont confrontés à la nécessité d'élargir l'accès aux études médicales à des étudiants issus de groupes ou communautés qui y sont peu représentés ${ }^{[9]}$. Le problème est dès lors de fonder les décisions d'admission sur la base de combinaisons complexes de critères tels que le mérite, l'équité, la responsabilité. Pour le dire autrement, il s'agit de faire en sorte que les décisions prises soient les plus justes, à la fois au sens de la justesse et de la justice. Dans une telle perspective, il faut alors bien percevoir, comme le rappelle fort opportunément le consensus de la Conférence d'Ottawa ${ }^{[9]}$, que les décisions en jeu ne se fondent pas sur des approches techniques mais sur un choix de valeurs dont plusieurs peuvent se révéler en conflit, et qu'elles devraient être explicitement traitées comme 
telles, en s'affranchissant de la conception erronée mais pourtant répandue que de tels choix seraient des procédures dégradées.

En conclusion, il n'est pas illégitime de chercher à documenter des aptitudes personnelles de la manière la plus précise possible, au regard de leur capacité à prédire une réussite ou à favoriser un profil professionnel qui serait désirable, mais il faut avoir à l'esprit les limites d'un tel projet ${ }^{[5]}$. Il est parallèlement devenu de plus en plus exigé que des critères en lien avec des valeurs sociales et politiques soient pris en compte. Dans un cas comme dans l'autre, il faut reconnaitre que tant les aptitudes individuelles que les valeurs sociales, qu'il conviendrait respectivement de prioriser, relèvent d'entités socialement construites, historiquement situées, qui devraient dès lors faire l'objet de débats et de choix démocratiquement négociés. Ainsi, la problématique de la sélection des étudiants pour les études de médecine doit bien instruire des questions d'ordre ontologique (quelle est la nature des attributs et des caractéristiques personnelles à considérer chez les étudiants ?). Elle doit aussi prendre position par rapport à des questions d'ordre épistémologique (s'agit-il de démontrer des résultats ou d'argumenter des processus ?). Elle doit enfin répondre à des questions d'ordre méthodologique (quels instruments de collecte de données faut-il utiliser ?). Mais il faut se convaincre profondément qu'il s'agit d'abord d'être en mesure de rendre explicite et de justifier publiquement au nom de quoi les décisions relatives au choix des étudiants sont prises. En cela, il s'agit bien, fondamentalement, d'une problématique de nature éthique.

Jean JOUQUAN

Rédacteur en chef

Mailto : jean.jouquan@chu-brest.fr

\section{Références}

1. Renaud J-S, Cantat A, Lakhal S, Bourget M, St-Onge C. Sélection des candidats en médecine : validité prédictive des Mini entrevues multiples en contexte francophone. Pédagogie Médicale 2016;17:7-21.
2. Frank JR, Snell LS, Sherbino J, Boucher A. Référentiel de compétences CanMEDS 2015 pour les médecins. Collège royal des médecins et chirurgiens du Canada, 2015.

3. Gustin M-P, Vinciguerra C, Isaac S, Burillon C, Etienne J. Approches d'apprentissage et réussite en première année commune des études de santé (PACES) en France. Pédagogie Médicale 2016;17: 23-43.

4. Jouquan J. Le choix des étudiants candidats aux études de médecine : enjeux sociaux et pédagogiques d'une décision académique. Pédagogie Médicale 2003;4: 5-8.

5. Patterson F, Knight A, Dowell J, Nicholson S, Cousans F, Cleland J. How effective are selection methods in medical education? A systematic review. Med Educ 2016;50:36-60.

6. Rees EL, Hawarden AW, Dent G, Hays R, Bates J, Hassell A. Evidence regarding the utility of multiple mini-interview (MMI) for selection to undergraduate health programs: a BEME systematic review: BEME Guide $N^{\circ}$ 37. Med Teach 2016;38:443-55

7. Fayolle A-V, Passirani C, Letertre E, Ramond A, Perrotin D, Saint-André J-P, Richard I. Sélection des étudiants en médicine : facteurs prédictifs de réussite ; une revue systématique de la littérature. Presse Med 2016;45:483-494.

8. Razack S, Lessard D, Hodges BD, Maguire MH, Steinert $\mathrm{Y}$. The more it changes; the more it remains the same: a foucauldian analysis of Canadian policy documents relevant to student selection for medical school. Adv Health Sci Educ 2014;19:161-181.

9. Prideaux D, Roberts C, Eva K, Centeno A, McCrorie P, McManus C, et al. Assessment for selection for the health care professions and specialty training: consensus statement and recommendations from the Ottawa 2010 Conference. Med Teach 2011;33: 215-23.

10. Boelen C, Woollard B. 2009. Social accountability and accreditation: A new frontier for educational institutions. Med Educ 43:887-894.

11. Cosnefroy L. L'apprentissage autorégulé, entre cognition et motivation. Grenoble : Presses Universitaires de Grenoble, 2011.

12. Berthoz A. La décision. Paris : Éd. Odile Jacob, 2003.

13. Tardif J, Dubois B. De la nature des compétences transversales jusqu'à leur évaluation : une course à obstacles, souvent infranchissables. Revue française de linguistique appliquée 2013;18:29-45. 
14. Parent F, Jouquan J. L'ambiguïté, les impasses et le discutable mérite du concept de compétence transversale. In : Parent F \& Jouquan J. Comment élaborer et analyser un référentiel de compétences en santé ? Une clarification conceptuelle et méthodologique de l'approche par compétences. Bruxelles : De Boeck Supérieur, 2015:189-92.

15. Urlings-Strop LC, Themmen APN, Stijnen T, Splinter TAW. Selected medical students achieve better than lottery-admitted students during clerkships. Med Educ 2011;45:1032-40.

16. Fauvet L, Jakoubovitch S, Mikol F. Profil et parcours des étudiants en première année commune aux études de santé. Études Résultats DREES (Direction de la Recherche, des Études, de l'Évaluation et des Statistiques), 2015.

17. Bourdieu P, Passeron JC. La Reproduction. Eléments pour une théorie du système d'enseignement. Paris : Éditions de Minuit, 1970.

18. Arrêté du 20 février 2014 relatif à l'expérimentation de nouvelles modalités d'admission dans les études médicales, odontologiques, pharmaceutiques et maïeutiques. Journal Officiel de la République Française, $n^{\circ} 0045$ du 22 février $2014: 186$. [On-line] Disponible sur : https://www.legifrance.gouv.fr/eli/ arrete/2014/2/20/ESRS1400114A/jo 